\title{
Comparative theoretical analysis of continuous wave laser cutting of metals at 1 and $10 \mu \mathrm{m}$ wavelength
}

\author{
Michael H. Brügmann • Thomas Feurer
}

Received: 9 September 2013/Accepted: 5 January 2014/Published online: 23 January 2014

(C) Springer-Verlag Berlin Heidelberg 2014

\begin{abstract}
We present a derivation and, based on it, an extension of a model originally proposed by V.G. Niziev to describe continuous wave laser cutting of metals. Starting from a local energy balance and by incorporating heat removal through heat conduction to the bulk material, we find a differential equation for the cutting profile. This equation is solved numerically and yields, besides the cutting profiles, the maximum cutting speed, the absorptivity profiles, and other relevant quantities. Our main goal is to demonstrate the model's capability to explain some of the experimentally observed differences between laser cutting at around 1 and $10 \mu \mathrm{m}$ wavelengths. To compare our numerical results to experimental observations, we perform simulations for exactly the same material and laser beam parameters as those used in a recent comparative experimental study. Generally, we find good agreement between theoretical and experimental results and show that the main differences between laser cutting with 1- and $10-\mu \mathrm{m}$ beams arise from the different absorptivity profiles and absorbed intensities. Especially the latter suggests that the energy transfer, and thus the laser cutting process, is more efficient in the case of laser cutting with $1-\mu \mathrm{m}$ beams.
\end{abstract}

\section{Introduction}

During the past years several theoretical and experimental studies investigating solid-state laser (i.e., disk or fiber

\author{
M. H. Brügmann $(\bowtie) \cdot$ T. Feurer \\ Institute of Applied Physics, University of Bern, Sidlerstrasse 5, \\ CH-3012 Bern, Switzerland \\ e-mail: michael.bruegmann@iap.unibe.ch \\ T. Feurer \\ e-mail: thomas.feurer@iap.unibe.ch
}

lasers operating around $1 \mu \mathrm{m}$ wavelength) inert gas fusion cutting have been carried out. Experiments revealed distinct differences with respect to the standard $\mathrm{CO}_{2}(10 \mu \mathrm{m})$ laser beam fusion cutting process. Specifically, the maximum speed for disk and fiber laser cutting of metal sheets is usually higher than for $\mathrm{CO}_{2}$ laser cutting given the same laser power output level [1]. Moreover, the high cut edge quality in $\mathrm{CO}_{2}$ laser cutting, with almost regular striation patterns, has not yet been achieved in fiber and disk laser cutting of workpieces with thicknesses greater than $5 \mathrm{~mm}$ [2]. Hitherto there is no rigorous explanation for these particular differences between $\mathrm{CO}_{2}$ and solid-state laser cutting.

In the past, theoretical analysis has focused on the wavelength-dependent absorptivity at the cutting front [8], the absorbed intensity [9], and the role of multiple reflections and its effects on the cutting front as well as on the cutting edges [12]. Also, hydrodynamic, mechanical, and thermal phenomena, such as the hydrodynamics of the melt layer [2-5], the melt removal from the cut kerf [6, 7], the effect of a high recoil pressure [10], and the temperature at the cutting front [11], were investigated in view of their influence on the cut quality.

Here, we analyze the differences between $\mathrm{CO}_{2}$ - and solid-state laser inert gas fusion cutting with the help of a model originally proposed by Niziev [13]. Our main goal is twofold. On the one hand, the numerical solutions in form of cutting and absorptivity profiles provide details on the energy transfer from the laser beam to the material and, thus, allow for a better understanding of the differences between $\mathrm{CO}_{2}$ and solid-state laser cutting. On the other hand, we compute solutions for same material and laser cutting parameters as used in a recent comparative experimental study [1], which allows for testing the prediction accuracy of the original and the modified Niziev model. 
This paper is organized as follows: in Sect. 2, we present a derivation of the Niziev equation based on local energy balance and show how it can be extended to incorporate heat conduction. Section 3 is devoted to the numerical solution and in Sect. 4 we summarize the parameters used in the numerical computations. In Sect. 5, the results in form of cutting and absorptivity profiles, maximum cutting speeds, and mean absorbed intensities are discussed. Additionally, the results are compared to experimental observations presented in reference [1]. Finally, in Sect. 6 we conclude by summarizing the main results.

\section{Theoretical modeling}

2.1 Niziev's model of continuous wave laser beam cutting of metals

In references [13, 14] Niziev and Nesterov presented a mathematical theory of continuous wave laser beam cutting. The model is based on a nonlinear first order partial differential equation that describes the shape of the cutting profile. The assumptions of the Niziev-Nesterov model are: (1) the process of removing molten material from the kerf takes place instantaneously; (2) the main function of the assist gas is to remove molten material; (3) chemical reactions, specifically oxidation of the molten material, are neglected; (4) heat conduction from the kerf to the bulk is not taken into account; (5) only a single reflection of the laser beam at the kerf wall is considered. In order to derive and, more importantly, to be able to extend the equation which governs the profile of the beam-material interaction front, we assume that the energy balance can be described locally and that the radiation field at the metal surface can be treated in the geometrical optics approximation. The latter condition restricts the application of the model to macroscopic variations of the surface profile, that is, the wavelength $\lambda$ of the radiation is small compared to the radius $R$ of the local surface curvature, i.e., $\lambda \ll R$. The former condition is fulfilled if the temperature distribution is localized within a small surface layer of depth $r_{\text {th }}$, i.e., $R \gg r_{\text {th }}=\chi / V_{0}$, where $\chi$ is the thermal diffusivity and $V_{0}$ is the cutting velocity. When appreciable values of the Peclet number $P e$ are reached, i.e., $P e=R \mathrm{~V}_{0} / \chi \gg 1$, this condition is always satisfied. In consequence of that, a high laser power, which leads to high values of $V_{0}$, ensures that the time-dependent surface profile

$h(x, y, z, t)=z-z(x, y, t)=0$

is governed by the local energy balance equation.

$f_{L} I \mathbf{e}_{S} \cdot \nabla h=\left(\mathbf{q}+\rho H \mathbf{V}_{0}\right) \cdot \nabla h$.
In Eq. (2) $f_{L} I \mathbf{e}_{S}\left(f_{L}\right.$ is the Fresnel absorption coefficient, $I$ denotes the laser intensity and $\mathbf{e}_{S}$ is the unit vector in the direction of the Poynting vector, i.e., $\mathbf{e}_{s}=\mathbf{S} /|\mathbf{S}|$ ) is the absorbed energy density flow due to the laser radiation, $\mathbf{q}$ is the heat flow, and $\rho H$ is the energy density necessary for melting and thus removing the irradiated material. Since for high values of $P e$ the conductive part $-\Lambda \nabla T$ of the heat flow is smaller than the convective part $\rho c\left(T_{m}-T_{0}\right) \mathbf{V}_{0}$, i.e., $\mathbf{q} \approx \rho c\left(T_{m}-T_{0}\right) \mathbf{V}_{0}$, the generalized ablation energy density is defined as

$\rho H^{\prime}=\rho c\left(T_{m}-T_{0}\right)+\rho H$,

where $H$ is the specific melt enthalpy, $T_{m}-T_{0}$ the difference between the melting temperature $T_{m}$ and the room temperature $T_{0}, c$ the specific heat, and $\rho$ the material density. It is worth pointing out that this definition of the generalized ablation energy density (Eq. (3)) implies that the process temperature is equal to the melting temperature $T_{m}$. With $\mathbf{V}_{0} \cdot \nabla h=-\partial h / \partial t$ Eq. (2) becomes

$\rho H^{\prime} \frac{\partial h}{\partial t}+f_{L} I \mathbf{e}_{S} \cdot \nabla h=0$.

After substituting Eq. (1) into Eq. (4) we obtain

$-\frac{\partial z}{\partial t}+\frac{f_{L}(\theta) I(x, y, z, t)}{\rho H^{\prime}}|\nabla h| \cos \theta=0$,

where $\cos \theta=\mathbf{e}_{S} \cdot(\nabla h /|\nabla h|)$. That is, $\theta$ is the local angle of incidence on the cutting surface, i.e., the angle between the unit Poynting vector $\mathbf{e}_{S}$ and the normal to the cutting surface. A schematic of the material coordinate system is depicted in Fig. 1 and the relevant angles are defined in Fig. 2. The axial shift of the focal position with respect to the workpiece is measured from the top surface of the material.

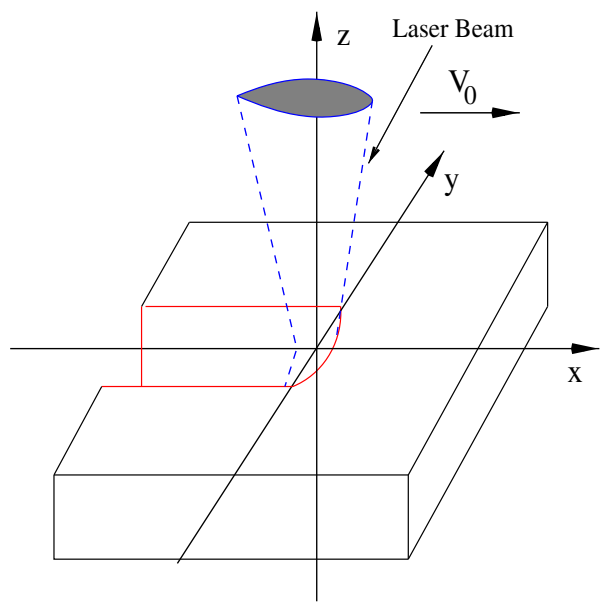

Fig. 1 The sample position and the beam propagation in the material coordinate system 
With this choice of coordinate system Eq. (5) takes the form

$$
\begin{aligned}
\frac{\partial z}{\partial t} \cos \alpha & \\
& +\frac{f_{L}\left(\theta, \beta-\beta_{0}\right) I\left(x-V_{0} t, y, z\right)}{\rho H^{\prime}} \cos \theta=0 .
\end{aligned}
$$

In Eq. (6) $\alpha=\arctan \sqrt{p^{2}+q^{2}}$, with $p=\partial z / \partial x$ and $q=\partial z / \partial y$, is the angle defined by $\cos \alpha=-(\nabla h /|\nabla h|)$. $\mathbf{z} /|\mathbf{z}|=-1 /|\nabla h|$. The angle $\beta=\arctan (q / p)$ is the angle between the incidence plane of the laser beam and its direction of movement. The Fresnel absorption coefficient as well as the angle $\beta_{0}$ are determined by the state of polarization of the incident light with respect to the cutting profile at $y=0$. For a plane polarized beam, the angle $\beta_{0}$ between the electric field vector and the beam velocity vector is constant and $f_{L}\left(\theta, \beta-\beta_{0}\right)$ is given by

$$
\begin{aligned}
f_{L}\left(\theta, \beta-\beta_{0}\right)= & f_{s}(\theta) \sin ^{2}\left(\beta-\beta_{0}\right) \\
& +f_{p}(\theta) \cos ^{2}\left(\beta-\beta_{0}\right),
\end{aligned}
$$

where $f_{s}(\theta)$ and $f_{p}(\theta)$ are the Fresnel formulae for $s$ - and $p$-wave absorption, respectively. From $y=0$ follows that the laser's plane of incidence coincides with its direction of movement, i.e., $\beta=0$. With that we obtain for $p-$, $s$-, and $c$-polarized light for which the angle $\beta_{0}$ in $f_{L}$ is

- $p$-polarization $\beta_{0}=0 \Rightarrow f_{L}(\theta, 0)=f_{p}(\theta)$,

- $s$-polarization $\beta_{0}=\pi / 2 \Rightarrow f_{L}(\theta, \pi / 2)=f_{s}(\theta)$,

- $c$-polarization $\beta_{0}=\pi / 4 \Rightarrow f_{L}(\theta, \pi / 4)=f_{c}(\theta)=1 / 2$ $\left(f_{p}(\theta)+f_{s}(\theta)\right)$

The expressions for the Fresnel absorption coefficients can be derived from Maxwell's equations (see for example [15-17]). For the case when $n^{2}+\kappa^{2} \gg 1$ (valid for metals at wavelength $\lambda>500 \mathrm{~nm}$ ) the Fresnel formulae for the $p$ - and $s$-polarizations are given by [17]

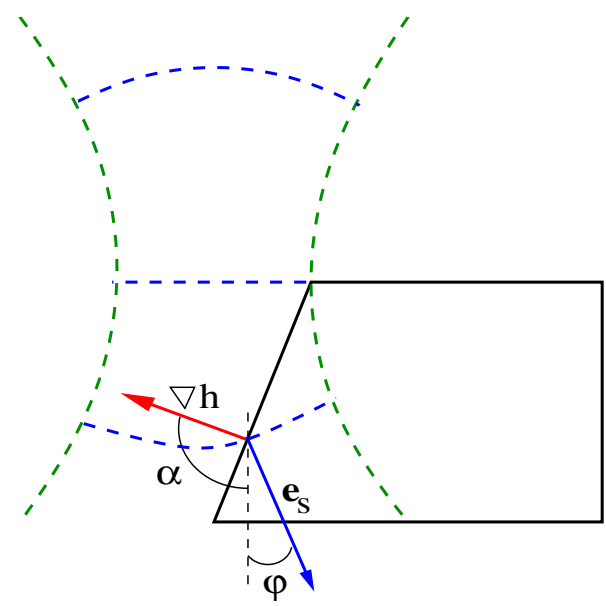

Fig. 2 Geometric parameters at the cutting front. Angle of incidence $\theta=\alpha+\varphi$ $f_{p}(\theta)=\frac{4 n \cos \theta}{\left(n^{2}+\kappa^{2}\right) \cos ^{2} \theta+2 n \cos \theta+1}$,

$f_{s}(\theta)=\frac{4 n \cos \theta}{n^{2}+\kappa^{2}+2 n \cos \theta+\cos ^{2} \theta}$,

where $n$ is the real and $\kappa$ the imaginary part of the refractive index. Equation (6) is fully equivalent to $\mathrm{Ni}$ ziev's Eqs. [13, 14]

$\frac{\partial z}{\partial t} \cos \alpha+\Psi(A)=0$

where $\Psi(A)$ is a function of the absorbed power modeling the threshold nature of material sublimation. The expression for $A$ reads

$A=I\left(x-V_{0} t, y, z\right) f_{L}\left(\theta, \beta-\beta_{0}\right) \cos \theta$.

The function $\Psi(A)$ is modeled by analytical dependence. In Niziev's paper most calculations were performed under the assumption that the function $\Psi(A)$ is a linear function of the quantity $A$, i.e., $\Psi(A)=K A$, with $K=1 /(\rho$ $c\left(T_{m}-T_{0}\right)+\rho H$ ) (note that Niziev uses $\rho H=\mu$ ).

The nonlinear partial differential Eq. (10) written in the laser's moving coordinate system, as defined in Fig. 3, reads

$$
\begin{aligned}
\frac{\partial z^{\prime}}{\partial t^{\prime}}= & V_{0} \frac{\partial z^{\prime}}{\partial x^{\prime}} \\
& -K I\left(x^{\prime}, y^{\prime}, z^{\prime}\right) f_{L}\left(\theta, \beta-\beta_{0}\right) \frac{\cos \theta}{\cos \alpha}
\end{aligned}
$$

The notations used throughout this paper are:

1. $(x, y, z, t)$ are the coordinates in the material reference frame;

2. $\left(x^{\prime}, y^{\prime}, z^{\prime}, t^{\prime}\right)$ are the coordinates in the moving beam reference frame;

3. $z_{, x}$ and $p$ denote the partial derivative $\frac{\partial z}{\partial x}$;

4. $z, y$ and $q$ denote the partial derivative $\frac{\partial z}{\partial y}$;

5. $z_{s}^{\prime}\left(x^{\prime}\right), z_{s}(x)$ denote the solutions of the stationary equations;

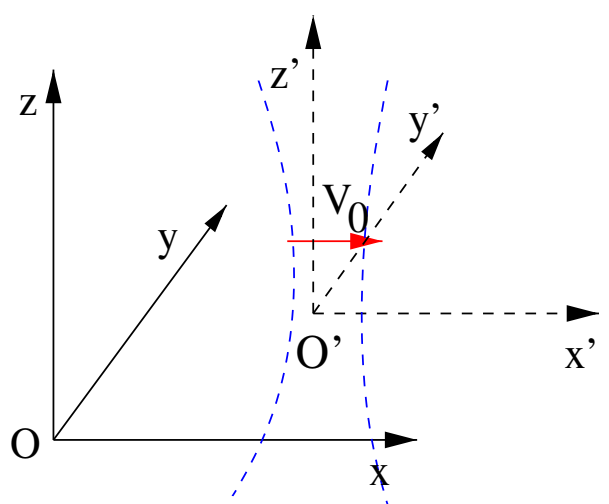

Fig. 3 Material and laser coordinate systems 
6. $z_{s, x}^{\prime}$ and $p_{s}^{\prime}$ denote the partial derivative $\frac{\partial z_{s}^{\prime}}{\partial x^{\prime}}$.

\subsection{Extended model}

Next, we extend Niziev's original model by abandoning the assumption that heat conduction from the kerf to the bulk can be neglected. The extended model accounts for heat conduction by adding an approximate expression for the conductive part $-\Lambda \nabla T$ of the heat flow

$-\Lambda \nabla T \approx \rho c\left(T_{m}-T_{0}\right)\left(\frac{P e}{2}\right)^{-0.64} \mathbf{V}_{0}$

which is derived from Eq. (13) in reference [18] and is based on a cylindrically shaped kerf. In order to introduce it in Niziev's basic equation it is necessary, for mathematical reasons, to assume that the heat flow is parallel to the cutting velocity $\mathbf{V}_{0}$. This introduces a maximum error of $36 \%$. For low Peclet numbers, which are found for thick workpieces, this error is partially compensated because Eq. (13) overrates the heat transport for $P e<0.2$. In other words, we expect better agreement with experimental results for thicker workpieces. After inserting Eq. (13) into the local energy balance, i.e., Eq. (2), we obtain instead of Eq. (3)

$$
\begin{aligned}
\rho H^{\prime}= & \rho c\left(T_{m}-T_{0}\right)\left[1+\left(\frac{P e}{2}\right)^{-0.64}\right] \\
& +\rho H .
\end{aligned}
$$

Equation (6) now needs to be solved considering Eq. (14) rather than Eq. (3). Generally, we expect that the absorptivity profiles and the absorbed intensities remain mostly unaffected by the extended model, however, the absorbed intensities now have to be partitioned between material removal and heat conduction. Thus, material removal and consequently the cutting speeds will most likely be lower.

\section{Numerical solutions}

In order to solve Eq. (12), Niziev split the function $z^{\prime}\left(x^{\prime}, y^{\prime}, t^{\prime}\right)$ into a stationary part $z_{s}^{\prime}\left(x^{\prime}, y^{\prime}\right)$ and a timedependent part $[13,14]$. The time dependence is treated as perturbation $\delta z^{\prime}\left(x^{\prime}, y^{\prime}, t^{\prime}\right)$ to the stationary part, i.e., $z^{\prime}\left(x^{\prime}, y^{\prime}, t^{\prime}\right)=z_{s}^{\prime}\left(x^{\prime}, y^{\prime}\right)+\delta z^{\prime}\left(x^{\prime}, y^{\prime}, t^{\prime}\right)$. The nonlinear partial differential equation for the stationary part is

$V_{0} \frac{\partial z_{s}^{\prime}}{\partial x^{\prime}}=K I\left(x^{\prime}, y^{\prime}, z_{s}^{\prime}\right) f_{L}\left(\theta_{s}, \beta_{s}-\beta_{0}\right) \frac{\cos \theta_{s}}{\cos \alpha_{s}}$.

The stationary solution $z_{s}^{\prime}\left(x^{\prime}, y^{\prime}\right)$ of Eq. (15) represents the shape of the cutting profile under stable cutting conditions. Generally, there are no exact solutions to the nonlinear differential equation and we have to resort to numerical methods. In order to extract the relevant parameters, we solve the model in three dimensions but set $y^{\prime}=0$ to access the relevant quantities at the cutting front. With this Eq. (15) becomes

$V_{0} \frac{\partial z_{s}^{\prime}}{\partial x^{\prime}}=K I\left(x^{\prime}, z_{s}^{\prime}\right) f_{L}\left(\theta_{s}, \beta_{0}\right) \frac{\cos \theta_{s}}{\cos \alpha_{s}}$,

with $\theta_{s}=\alpha_{s}+\varphi_{s}, \alpha_{s}=\arctan \left(\frac{\partial z_{s}^{\prime}}{\partial x^{\prime}}\right), \beta_{s}=0$, and $\varphi_{s}=$ $\varphi_{s}\left(x^{\prime}, z_{s}^{\prime}\right)$. Equation (16) is a nonlinear implicit ordinary differential equation of first order and can be solved by the method of characteristics [13, 14]. In the process, Eq. (16) is transformed to a system of ordinary differential equations (ODEs) [19],

$$
\begin{aligned}
\frac{d x^{\prime}}{d t^{\prime}}= & 1-\frac{K I\left(x^{\prime}, z_{s}^{\prime}\right)}{V_{0} \cos \alpha_{s}}\left[f_{L, \theta_{s}} \theta_{s, p_{s}^{\prime}} \cos \theta_{s}+f_{L} \cos \theta_{s} \tan \alpha_{s} \alpha_{s, p_{s}^{\prime}}\right. \\
& \left.-f_{L} \sin \theta_{s} \theta_{s, p_{s}^{\prime}}\right],
\end{aligned}
$$

$$
\begin{aligned}
\frac{d z_{s}^{\prime}}{d t^{\prime}}= & p_{s}^{\prime}-p_{s}^{\prime} \frac{K I\left(x^{\prime}, z_{s}^{\prime}\right)}{V_{0} \cos \alpha_{s}}\left[f_{L, \theta_{s}} \theta_{s, p_{s}^{\prime}} \cos \theta_{s}\right. \\
& \left.+f_{L} \cos \theta_{s} \tan \alpha_{s} \alpha_{s, p_{s}^{\prime}}-f_{L} \sin \theta_{s} \theta_{s, p_{s}^{\prime}}\right],
\end{aligned}
$$$$
\frac{d p_{s}^{\prime}}{d t^{\prime}}=\frac{K}{V_{0} \cos \alpha_{s}}\left[I_{, x^{\prime}} f_{L} \cos \theta_{s}+I\left(x^{\prime}, z_{s}^{\prime}\right) f_{L, \theta_{s}} \theta_{s, x^{\prime}} \cos \theta_{s}\right.
$$

$$
\begin{aligned}
& \left.-I\left(x^{\prime}, z_{s}^{\prime}\right) f_{L} \sin \theta_{s} \theta_{s, x^{\prime}}\right] \\
+ & p_{s}^{\prime} \frac{K}{V_{0} \cos \alpha_{s}}\left[I_{z_{s}^{\prime}} f_{L} \cos \theta_{s}+I\left(x^{\prime}, z_{s}^{\prime}\right) f_{L, \theta_{s}} \theta_{s, z_{s}^{\prime}} \cos \theta_{s}\right. \\
& \left.-I\left(x^{\prime}, z_{s}^{\prime}\right) f_{L} \sin \theta_{s} \theta_{s, z_{s}^{\prime}}\right] .
\end{aligned}
$$

The explicit expression for $\varphi_{s}$ reads

$$
\begin{aligned}
& \varphi_{s}= \\
& \arctan \left[\frac{2 k x^{\prime} z_{s}^{\prime}\left(z_{s}^{\prime 2}+z_{r}^{\prime 2}\right)}{\left[2 k\left(z_{s}^{\prime 2}+z_{r}^{\prime 2}\right)^{2}-2\left[M^{2}\right] z_{r}^{\prime}\left(z_{s}^{\prime 2}+z_{r}^{\prime 2}\right)-k x^{\prime 2}\left(z_{s}^{\prime 2}-z_{r}^{\prime 2}\right)\right]}\right],
\end{aligned}
$$

where $k=\frac{2 \pi}{\lambda}, z_{r}^{\prime}$ is the Rayleigh length, and $\left[M^{2}\right]$ is the integer closest to the beam quality factor $M^{2}$. The system of ODEs (17)-(19) was numerically solved with the help of MATLAB (ode45 with absolute and relative error tolerances set to $10^{-12}$ and $10^{-9}$ ). It is worthwhile to point out that the numerical solutions of the system of ODEs 
(17)-(19) become numerically very unstable when the value of the angle $\alpha_{s}$ approaches $\pi / 2$. This problem can be fixed by adding a small quantity $\delta \approx 10^{-7}$ to $\alpha_{s}$. In order to control the accuracy of the computed solutions, we substituted the computed values for $x^{\prime}, z^{\prime}{ }_{s}$ and $p^{\prime}{ }_{s}$ into Eq. (16). With this the equation for the maximal absolute error of the computations reads

$$
\begin{aligned}
\max \left|\varepsilon_{\mathrm{rr}}\right|= & \max \mid \mathrm{V}_{0} \mathrm{p}_{\mathrm{s}(\text { Calc. })}^{\prime} \\
& -K I\left(x_{(\text {Calc. })}^{\prime}, z_{s(\text { Calc. })}^{\prime}\right) f\left(\theta_{s(\text { Calc. })}\right) \frac{\cos \theta_{s(\text { Calc. })}}{\cos \alpha_{s(\text { Calc. })}} \mid,
\end{aligned}
$$

where $x_{(\text {Calc. })}^{\prime}, z_{s(\text { Calc. })}^{\prime}, p_{s(\text { Calc. })}^{\prime}, \theta_{s(\text { Calc. })}$ and $\alpha_{s(\text { Calc. })}$ denote the computed values. In all simulations shown hereafter the maximal absolute error was always $\max \left|\varepsilon_{\mathrm{rr}}\right| \leq 10^{-4}$.

\section{Model parameters}

In order to compare the numerical solutions to a recently published comparative experimental study, we performed the computations for the laser cutting parameters and material parameters as given in reference [1]. In this reference a $\mathrm{CO}_{2}$ laser and a disk laser were used to cut coldwork tool steel 90MnCrV8 (AISI O2) plates with thicknesses of 5 and $8 \mathrm{~mm}$. All experiments were carried out with an average laser power of $3 \mathrm{~kW}$. We approximate both laser beams by Super-Gauss beams

$I(x, y, z)=\frac{I_{0}}{l(z)} \exp \left[-2\left|\frac{\sqrt{\left(x^{2}+y^{2}\right)}}{w_{0} l(z)^{\frac{1}{2}}}\right|^{2 m}\right]$,

where $m$ is the Super-Gauss order, $w_{0}$ the waist radius, and $l(z)=1+\left(z / z_{r}\right)^{2}$ with the Rayleigh length $z_{r}=(\pi$ $\left.W_{0}^{2}\right) /\left(M^{2} \lambda\right)$ where $M^{2}$ is the beam quality factor and $W_{0}$ is the real-beam size. The waist radius $w_{0}$ and the real-beam size $W_{0}$ are related through [20],

$W_{0}^{2}=w_{0}^{2} \frac{2^{\left(2-\frac{1}{m}\right)}}{3} \frac{\Gamma\left(1+\frac{3}{2 m}\right)}{\Gamma\left(1+\frac{1}{2 m}\right)}$.

The beam quality factor $M^{2}$ of a Super-Gauss beam of the order $m$ can be calculated from [20]

$M^{4}=\left(\frac{2 m}{3}\right) \frac{\Gamma\left(2-\frac{1}{2 m}\right) \Gamma\left(1+\frac{3}{2 m}\right)}{\left[\Gamma\left(1+\frac{1}{2 m}\right)\right]^{2}}$.

For both lasers the beam parameters $m$ and $M^{2}$ were obtained through a fit of the Super-Gauss beam to the experimental data. The measured beam profile of the $\mathrm{CO}_{2}$ was almost perfectly Gaussian with $M^{2}=1.36$. To determine $m$ for $M^{2}=1.36$, we solve Eq. (24) for this value of
$M^{2}$ and the graphical solution results in $m=2.86$. Since $m$ here is an integer we choose the closest integer, in this case $m=3$. By inserting $m=3$ into Eq. (24) we obtain a corresponding quality factor $M^{2}=1.39$. Then we use the measured real-beam radius $W_{0}$ to determine the beam waist $w_{0}$ and the Rayleigh length $z_{r}$. For the disk laser the measured beam profile was more top-hat like with a quality factor of $M^{2}=14.30$. In this case, we derive from Eq. (24) $m=307.3$ and the closest integer is $m=307$. The measured and the fitted values are summarized in Table 1.

Both lasers emitted unpolarized beams, that is, we use the Fresnel coefficient for a $c$-polarized beam. The material constants for the cold-work steel $90 \mathrm{MnCrV} 8$ workpiece are listed in Table 2.

The refractive index $n$ and the extinction coefficient $\kappa$ are estimated with the help of Drude's theory [8], by calculating an average electron density from the stoichiometric mixture of elements.

\section{Results and discussion}

A large number of publications, e.g., [21-23], experimentally investigate $\mathrm{CO}_{2}$ laser cutting at $10.6 \mu \mathrm{m}$ and more recent publications $[1,7,10,24]$, due to the emergence of high-power fiber and disk lasers, started to present results at $1.03 \mu \mathrm{m}$. Here, we compare our theoretical model to experiments published on a comparative study of inert gas fusion cutting of 5- and 8-mm-thick 90MnCrV8 workpieces at 1.03 and $10.6 \mu \mathrm{m}$, respectively [1]. Specifically, we

Table 1 Measured beam parameters of the $\mathrm{CO}_{2}$ and the disk laser taken from reference [1]. The fitted values represent the best approximation of a Super-Gaussian beam to the experimental data

\begin{tabular}{lllll}
\hline Laser source & $m$ & $M^{2}$ & $W_{0}(\mu \mathrm{m})$ & $z_{r}(\mathrm{~mm})$ \\
\hline $\mathrm{CO}_{2}$ (exp) & & 1.36 & 76.123 & 1.27 \\
$\mathrm{CO}_{2}$ (fit) & 3 & 1.39 & 76.123 & 1.24 \\
Disk (exp) & & 14.30 & 76.171 & 1.20 \\
Disk (fit) & 307 & 14.29 & 76.171 & 1.24 \\
\hline
\end{tabular}

Table 2 Material constants for the $90 \mathrm{MnCrV} 8$ workpiece

\begin{tabular}{lll}
\hline Material constant & Symbol & Value \\
\hline Density & $\rho$ & $7600 \mathrm{~kg} / \mathrm{m}^{3}$ \\
Specific heat & $c$ & $460 \mathrm{~J} /(\mathrm{kg} \mathrm{K})$ \\
Melting temperature & $T_{m}$ & $1753.15 \mathrm{~K}$ \\
Latent heat of melting & $H$ & $250 \mathrm{~J} / \mathrm{g}$ \\
Index of refraction $(1.03 \mu \mathrm{m})$ & $n$ & 7.24 \\
& $\kappa$ & 4.24 \\
Index of refraction $(10.6 \mu \mathrm{m})$ & $n$ & 20.77 \\
& $\kappa$ & 19.67 \\
\hline
\end{tabular}


(a)

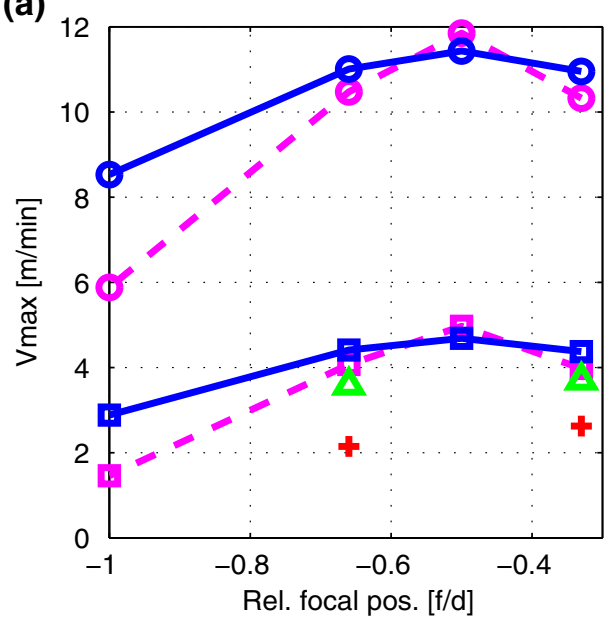

Fig. 4 Maximum cutting speeds and mean absorbed intensities for the 5-mm-thick workpiece. a Maximum cutting speed as function of the focal position for a $5 \mathrm{~mm}$ thick workpiece. Magenta dashed curves $\mathrm{CO}_{2}$-laser, blue solid curve disk laser, circles basic model, squares extended model. The ex-perimental values from [1] for the

(a)

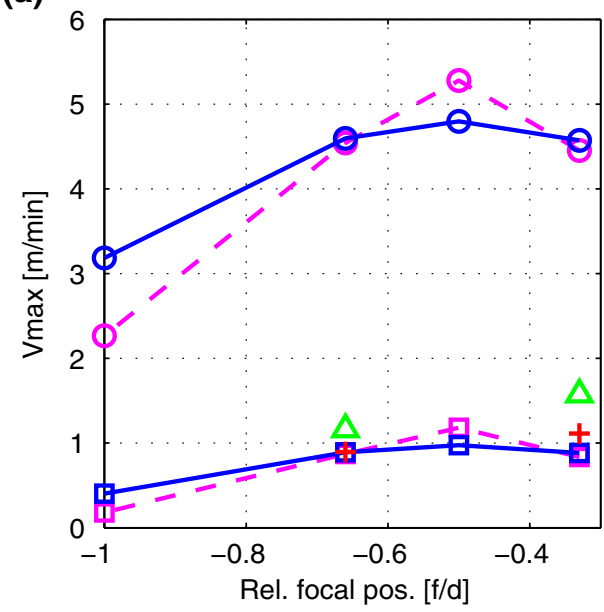

Fig. 5 Maximum cutting speeds and mean absorbed intensities for the 8-mm-thick workpiece. a Maximum cutting speed as function of the focal position for a $8 \mathrm{~mm}$ thick workpiece. Magenta dashed curve $\mathrm{CO}_{2}$-laser, blue solid curve disk laser, circles basic model, squares extended model. The experimental values from [1] for the $\mathrm{CO}_{2}$-laser

investigate the maximum cutting speed as a function of focus position with respect to the workpiece and the thickness of the workpiece and analyze the shape of the cutting profile. The maximum cutting speed is defined as the speed for which the calculated cutting profile is just deep enough to cut through a workpiece of a given thickness.

\subsection{Maximum cutting speed}

The main observations in reference [1] with respect to the maximum cutting speed are that it is generally higher for

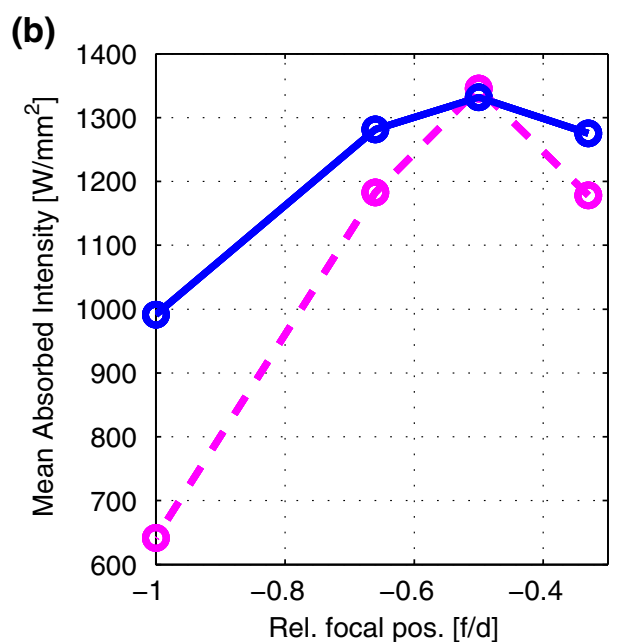

$\mathrm{CO}_{2}$-laser (red cross) and the disk laser (green triangles) are shown for reference. b Mean absorbed intensity as function of the focal position for a $5 \mathrm{~mm}$ thick workpiece calculated from the extended model. Magenta dashed curve $\mathrm{CO}_{2}$-laser, blue solid curve disk laser

(b)

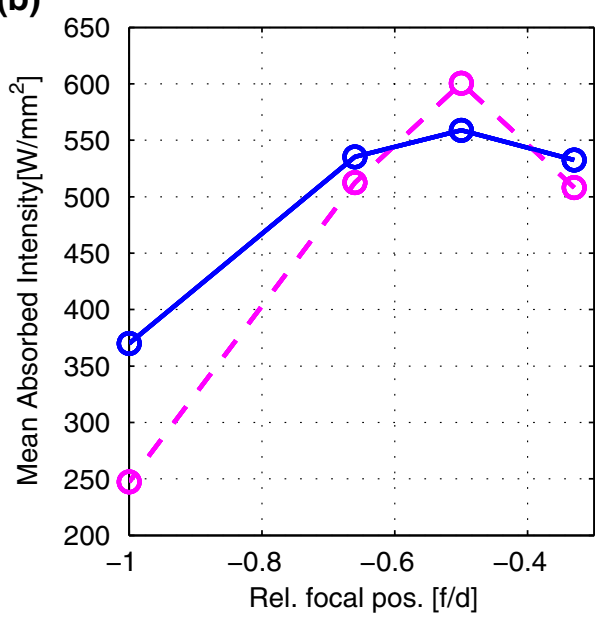

(red cross) and the disk laser (green triangles) are shown for reference. $\mathbf{b}$ Mean absorbed intensity as function of the focal position for a $8 \mathrm{~mm}$ thick workpiece calculated from the extended model. Magenta dashed curve $\mathrm{CO}_{2}$-laser, blue solid curve disk laser

the disk laser, i.e., for wavelengths around $1 \mu \mathrm{m}$, decreases with material thickness, and depends on the position of the beam focus with respect to the material position. Figures 4 and 5 show the simulated cutting speed as a function of the relative focal position $\mathrm{fld}$ for a 5 - and a 8-mm-thick workpiece. The upper surface corresponds to $f / d=0$ and the lower surface to $f / d=-1$, respectively.

We start with the original Niziev model. An important result is that the highest cutting speeds for the disk as well as for the $\mathrm{CO}_{2}$ laser are achieved when the focal position is at the middle of the workpiece, i.e., for $f / d \approx-0.5$ (see 
Figs. 4a, 5a). Further, the maximum cutting speed for the disk laser is almost always larger than for the $\mathrm{CO}_{2}$ laser (see Figs. 4a, 5a). If the focal position is at the middle of the workpiece our computations indicate a higher maximum cutting speed for the $\mathrm{CO}_{2}$-laser. All of these results are in qualitative agreement with the experiments (green and red symbols in Figs. 4a, 5a); however, the quantitative agreement is rather poor. For example, our theoretical maximum cutting speeds for the focal positions $f / d=$ -0.33 and $f / d=-0.66$ are by a factor of $2-5$ higher than the experimental values. Despite the differences in absolute values it is worthwhile noting that the ratio of the maximum cutting speeds of the two laser sources is between one and two, which is in good agreement with the experimental observations. We attribute the quantitative differences to mainly two effects. First, the refractive index and the extinction coefficient were estimated by means of Drude's theory. Since the $90 \mathrm{MnCrV} 8$ workpiece is an alloy, the values for $n$ and $\kappa$ as obtained from Drude's theory are only approximately correct. Second, the ejected molten layer from the cutting front and the heat conductivity in the bulk material are neglected. They affect the energy balance since they both transport energy away from the kerf. Both reduce the amount of energy disposable for the melting process and, as a consequence, less melt is produced per unit time and the cutting speed is reduced.

Next, we use the extended model, that is, we include heat conduction to the bulk material. For comparison, these simulations are also shown in Figs. 4a and 5a. Obviously the net effect of the heat conductivity to the bulk is a lowering of the cutting speed for both lasers by a factor between 3 and 5. That is, by including heat conduction the quantitative agreement between the model and the experimental observations improves and is excellent especially for the 8-mm-thick workpiece. For the 5-mm-thick workpiece, we find that some of the computed cutting speeds are somewhat higher than the measured ones; nevertheless, the agreement is substantially better than for the basic model.

In order to analyze the effectiveness of the energy transfer from the laser beam to the workpiece, we computed the mean absorbed intensity $\langle I\rangle$. It is obtained by dividing the line integral of the absorbed intensity along the cutting profile by the length of the cutting profile, i.e.,

$$
<I>=\frac{\int_{x_{0}^{\prime}}^{x_{f}^{\prime}} I\left(x^{\prime}, z_{s}^{\prime}\right) f_{c}\left(\theta_{s}\right) \cos \theta_{s} \sqrt{1+\left(\frac{d z_{s}^{\prime}}{d x^{\prime}}\right)^{2}} d x^{\prime}}{\int_{x_{0}^{\prime}}^{x_{f}^{\prime}} \sqrt{1+\left(\frac{d z_{s}^{\prime}}{d x^{\prime}}\right)^{2}} d x^{\prime}} .
$$

The mean absorbed intensities as function of the focal position are depicted in Figs. $4 \mathrm{~b}$ and $5 \mathrm{~b}$ and the general dependence is similar for both lasers. For focal positions closer to the top or the bottom surface of the workpiece, the mean absorbed intensity for the disk laser is always higher. This is the main reason why the cutting speeds for the disk laser are larger than the corresponding cutting speeds for the $\mathrm{CO}_{2}$ laser. Only for focal positions located around the middle of the workpiece the absorbed intensity can be higher for the $\mathrm{CO}_{2}$ laser. Comparing the curves of the mean absorbed intensities to the corresponding curves of the maximum cutting speeds suggests, not surprisingly, that the cutting speeds are directly proportional to the mean absorbed intensities. It is worthwhile mentioning that the mean absorbed intensities are virtually identical in both models.

Figure 6a shows the computed maximum cutting speed as a function of workpiece thickness when the focal position is at the middle of the workpiece. The model predicts a higher performance of the disk laser beam in the thickness range up to $5 \mathrm{~mm}$, as can be seen by inspecting the difference in cutting speeds shown in Fig. 6b. This advantage in performance disappears for thicker workpieces. Both
Fig. 6 Maximum cutting speed as function of the workpiece thickness. a Maximum cutting speed as function of the workpiece thickness. Focal position $f / d=-0.50$. Magenta pecked curve $\mathrm{CO}_{2}$-laser, blue solid curve disk laser, circles basic model, squares extended model. b Difference of the maximum cutting speed as function of the workpiece thickness. Focal position $f / d=$ -0.50 . Red solid line,circles basic model, green pecked line, squares extended model (a)

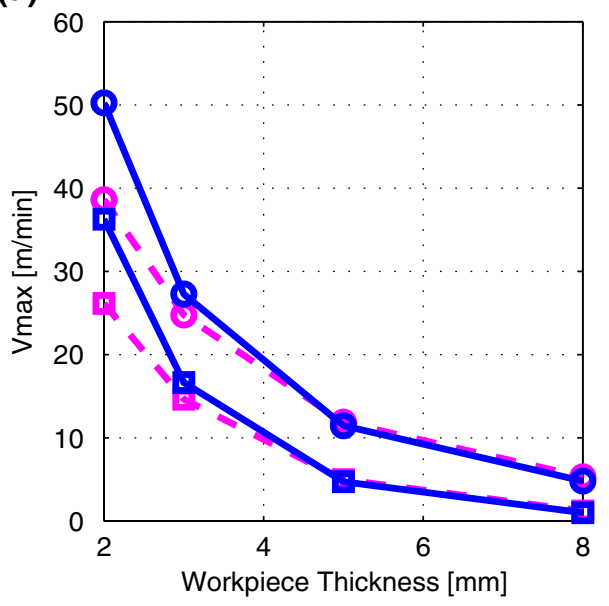

(b)

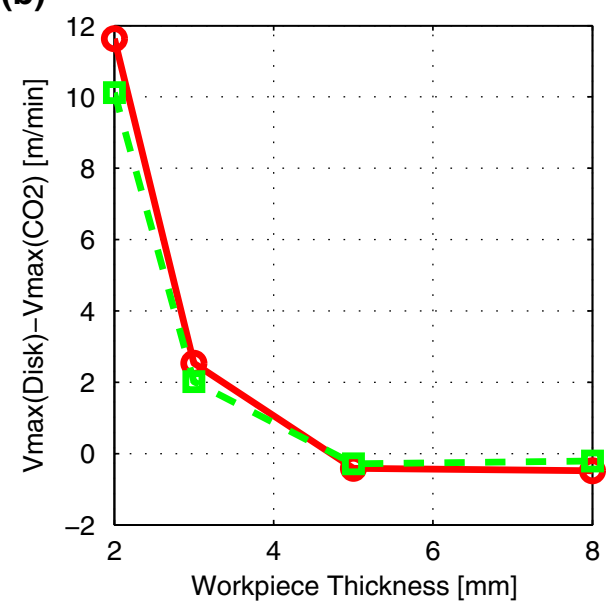


findings are in good agreement with the experiments (see Ref. [24] and references therein). Comparing the results of the basic and the extended model shows the same overall trend (see Fig. 6b), however, the absolute cutting speeds resulting from the extended model are smaller (see Fig. 6a).

The different results from the two models, specifically with respect to the maximum cutting speed, suggest that heat conduction losses are substantial and must not be neglected. Figure $7 \mathrm{a}, \mathrm{c}$ show the heat conduction flux and Fig. $7 \mathrm{~b}, \mathrm{~d}$ the heat conduction flux relative to the absorbed intensity for the 5- and 8-mm-thick workpieces.

The conductive losses with respect to the absorbed intensities are substantial for both lasers irrespective of workpiece thickness. For example, for an 8-mm-thick workpiece over $80 \%$ of the absorbed intensity is removed through heat conduction and does not contribute to the removal of material. The conductive losses are almost always larger for the $\mathrm{CO}_{2}$ laser than they are for the disk laser. Only when the focal position is around the middle of the workpiece, i.e., $f l d \approx-0.5$, the situation is reversed. Also, the conductive losses increase with decreasing cutting speed which is a consequence of the dependence of the generalized ablation energy density, see Eq. (14), on the Peclet number. Since the absorbed laser intensity is only a fraction of the incoming laser intensity the conductive losses for the 5-mm-thick workpiece, when compared to the incoming intensity, are between 21 and $33 \%$ for the $\mathrm{CO}_{2}$ laser and between 28 and $36 \%$ for the disk laser. In the case of the 8 -mm-thick workpiece the corresponding losses lie between 10 and $20 \%$ for the $\mathrm{CO}_{2}$ laser and between 15 and $20 \%$ for the disk laser. These values are in good agreement with the conductive losses reported in references [11] and [25].

\subsection{Cutting profiles}

In this subsection, we compare the computed cutting and absorptivity profiles for a 5-mm-thick $90 \mathrm{MnCrV} 8$-workpiece to the experimental results in reference [1].
Fig. 7 Heat conduction losses for the 5- and 8-mm-thick workpieces. a Heat conduction losses as function of the focal position for a $5 \mathrm{~mm}$ thick workpiece. Magenta dashed curves $\mathrm{CO}_{2}$-laser, blue solid curve disk laser. b The conductive losses relative to the absorbed intensities as function of the focal position for a $5 \mathrm{~mm}$ thick workpiece. Magenta dashed curve $\mathrm{CO}_{2}$-laser, blue solid curve disk laser. c Heat conduction losses as function of the focal position for a $8 \mathrm{~mm}$ thick workpiece. Magenta dashed curves $\mathrm{CO}_{2}$-laser, blue solid curve disk laser. d The conductive losses relative to the absorbed intensities as function of the focal position for a $8 \mathrm{~mm}$ thick workpiece. Magenta dashed curve $\mathrm{CO}_{2}$-laser, blue solid curve disk laser (a)

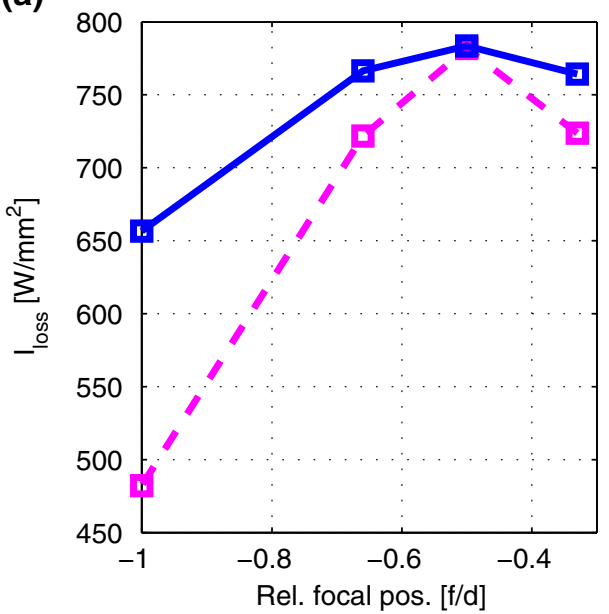

(b)

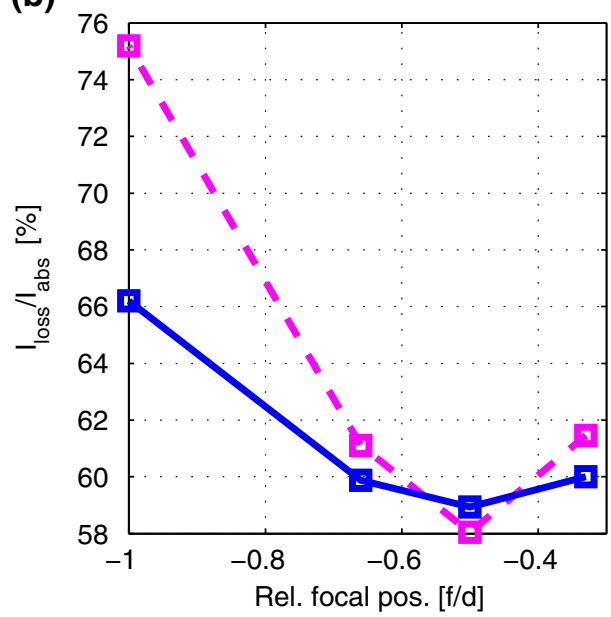

(c)

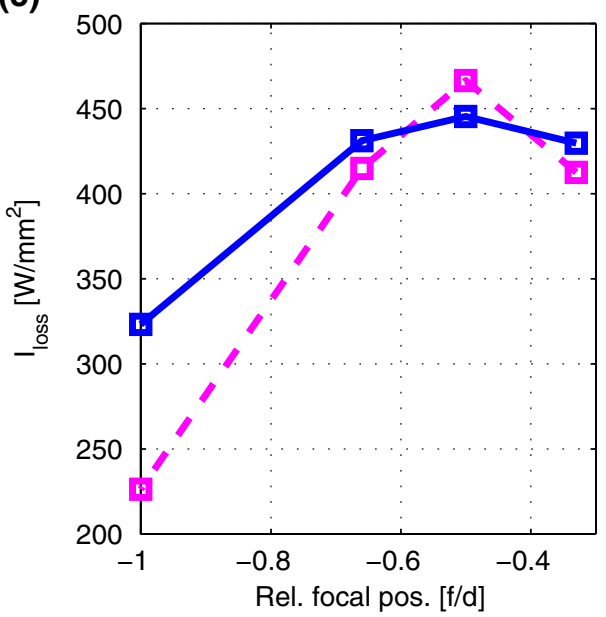

(d)

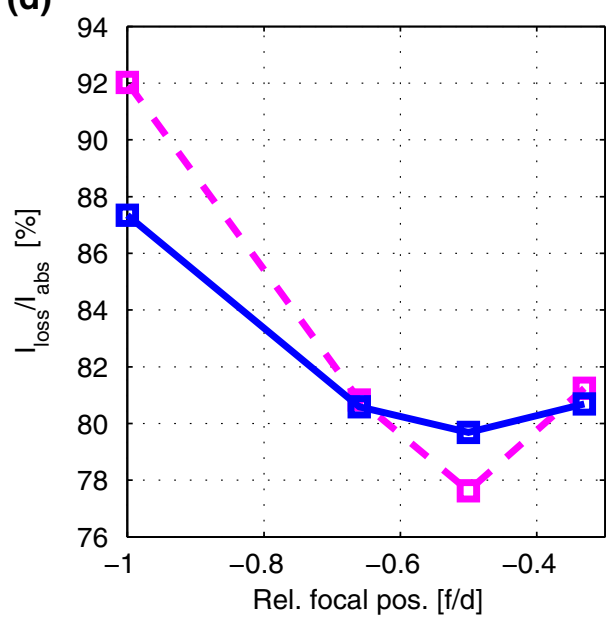


Fig. 8 Cutting profiles for a 5-mm-thick 90MnCrV8 workpiece. a Cutting profiles. Focal position $f / d=-0.33$. Magenta pecked curve $\mathrm{CO}_{2-}$ laser, blue solid curve disk laser. The red lines mark the top and bottom edges of the workpiece. b Cutting profiles. Focal position $f / d=-0.66$. Magenta pecked curve $\mathrm{CO}_{2}$-laser, blue solid curve disk laser. The red lines mark the top and bottom edges of the workpiece (a)

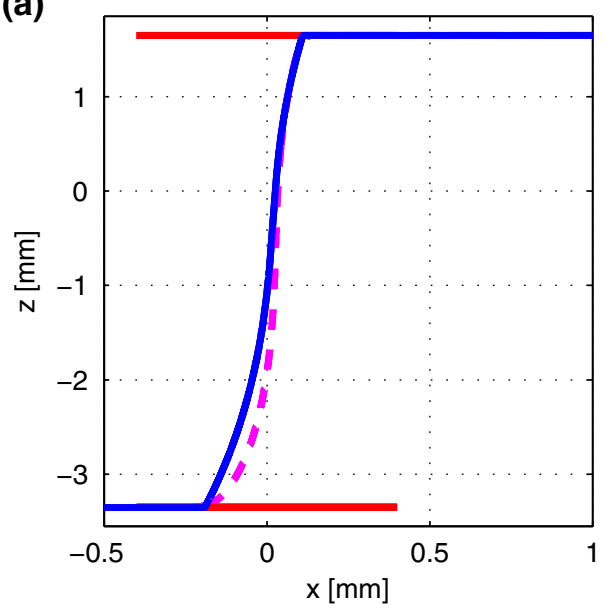

(b)

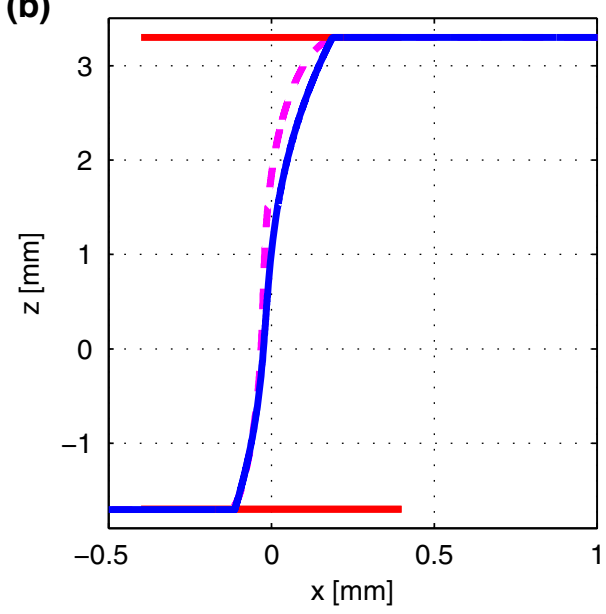

Figure $8 \mathrm{a}, \mathrm{b}$ show the calculated cutting profiles for the focal positions $f / d=-0.33$ and $f / d=-0.66$, respectively.

By visual inspection of the shape of the computed and the measured cutting profiles as presented in reference [1], we find a very good qualitative agreement. Figure $8 \mathrm{a}, \mathrm{b}$ also suggest that for a focal position of $f / d=-0.33$ the cutting profiles for disk and $\mathrm{CO}_{2}$-laser differ more on the lower part of the workpiece while for the $f / d=-0.66$ the differences are more pronounced at the upper part of the workpiece. In order to compare the calculated cutting profiles to the measured ones in a more quantitative way, we determine the projected longitudinal extension of the cutting profile $\Delta L$. It is defined as (see also reference [1]) the distance between the start point on the top surface and the end point on the bottom surface of the cutting profile. The experimental and the computed values for $\Delta L$ are summarized in Table 3.

While the computed values $\Delta L$, on an absolute scale, are too low by about a factor of two, the computed ratio $\Delta L_{\mathrm{CO}_{2}} / \Delta L_{\text {disk }}$ is in excellent agreement with the experimental result.

There are several reasons which might be responsible for the discrepancies observed when comparing absolute numbers. First, there are experimental uncertainties, e.g., the cutting profile after switching off the laser and the gas flow might not be identical to the one during the cutting process. Second, the model is based on several approximations which affect the cutting profile more than other parameters, for example, the influence of multiple

Table 3 Projected longitudinal extension of the cutting profile for a focal position $f / d=-0.33$

\begin{tabular}{lll}
\hline Laser & $\Delta L(\mathrm{~mm})$ theory & $\Delta L(\mathrm{~mm})$ experiment \\
\hline $\mathrm{CO}_{2}$ & $\sim 0.421$ & $\sim 0.750$ \\
Disk & $\sim 0.301$ & $\sim 0.500$ \\
Ratio & $\sim 1.40$ & $\sim 1.50$ \\
\hline
\end{tabular}

reflections. Experimental investigations have shown that for thicker materials and a cutting speed close to the maximum value, the effect of the multiple reflections on the absorptivity becomes important at the bottom part of the cutting front [26].

Based on the cutting profiles, we next analyze the absorptivity profiles as shown in Fig. 9a, b. For a focal position of $f l d=-0.33$, the minima of the absorptivity curves for the $\mathrm{CO}_{2}$ and disk laser are located in the upper part of the workpiece. In this case, the absorptivity for the $\mathrm{CO}_{2}$ laser is larger than the absorptivity for the disk laser along most parts of the cutting profile. Only at the top and the bottom part of the cutting profile the situation is reversed. For a focal position of $f / d=-0.66$ the absorptivity profiles are very similar, however, the curves are shifted towards the bottom surface of the workpiece. The minima of the computed absorptivity curves for both lasers are found to coincide approximately with the position of the beam focus. The same general behavior can be observed in the experimental absorptivity curves as shown in Fig. 9 of reference [1]. The experimental absorptivity curve for the disk laser also shows a minimum approximately at the middle of the workpiece. The experimental curve for the $\mathrm{CO}_{2}$ laser shows minima towards the upper and lower part of the workpiece, but also a minimum at about a depth of $3 \mathrm{~mm}$. The high-frequency fluctuations in the experimental absorptivity curves are most likely due to hydrodynamic instabilities. Since the Niziev model assumes that the molten material is removed instantaneously from the kerf, those fluctuations cannot be reproduced by the computed curves. Overall however, we find a good agreement between the theoretical and experimental absorptivity curves. By comparing the absorptivity profiles with the corresponding angle of incidence profiles, as shown in Fig. 9c, d, we find that the maxima of the absorptivity profiles are located in regions of the cutting front where the incidence angle is comparable to the Brewster angle. 
Fig. 9 Absorptivity and angle of incidence profiles for a 5-mm-thick 90MnCrV8 workpiece. a Absorptivity profiles. Focal position $f / d=$ -0.33. Magenta pecked curve $\mathrm{CO}_{2}$-laser, blue solid curve disk laser. b Absorptivity profiles. Focal position $f / d=-0.66$. Magenta pecked curve $\mathrm{CO}_{2^{-}}$ laser, blue solid curve disk laser. c Angle of incidence. Focal position $f / d=-0.33$. Magenta pecked curve $\mathrm{CO}_{2}$-laser, blue solid curve disk laser. d Angle of incidence. Focal position $f l d=-0.66$. Magenta pecked curve $\mathrm{CO}_{2}$-laser, blue solid curve disk laser (a)

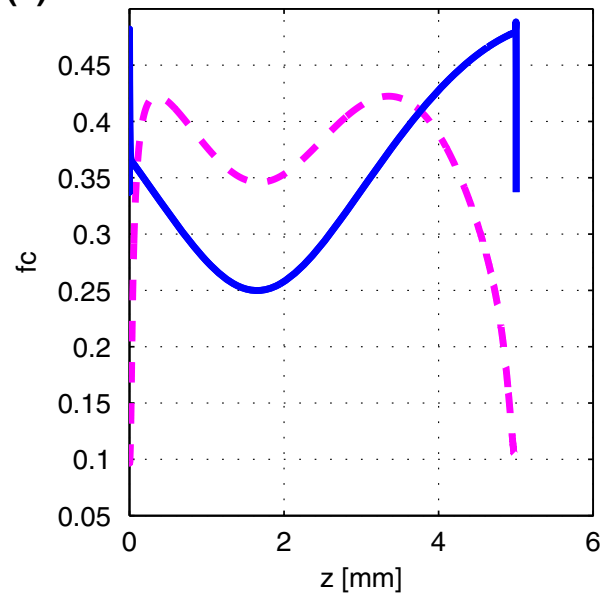

(c)

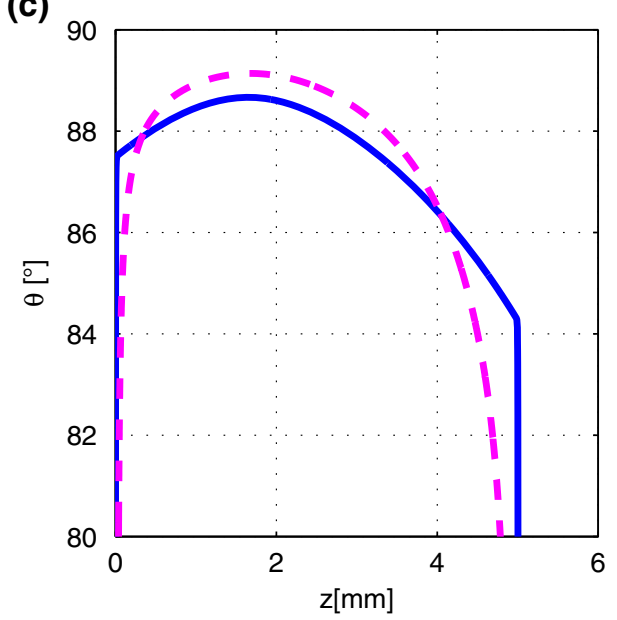

(a)

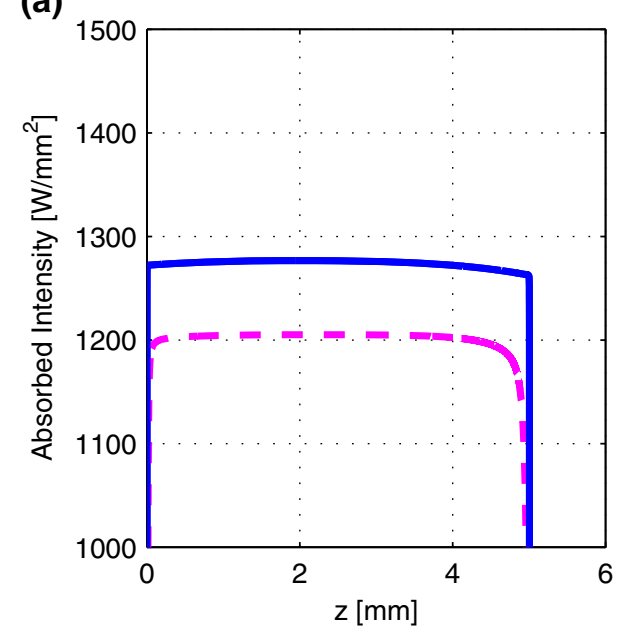

(b)

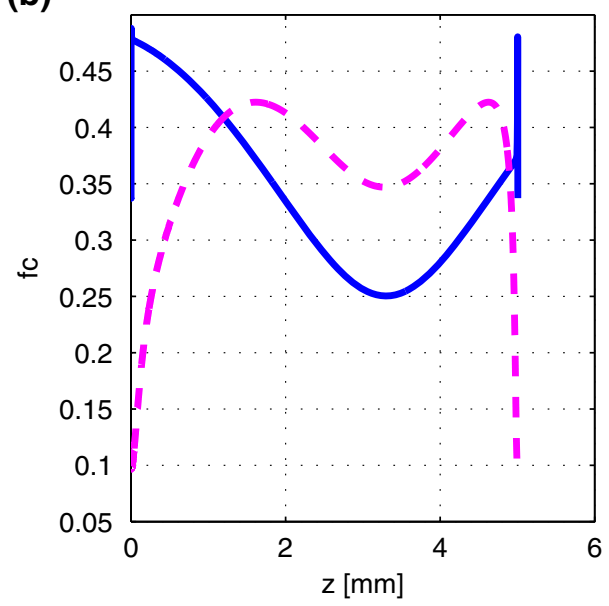

(d)

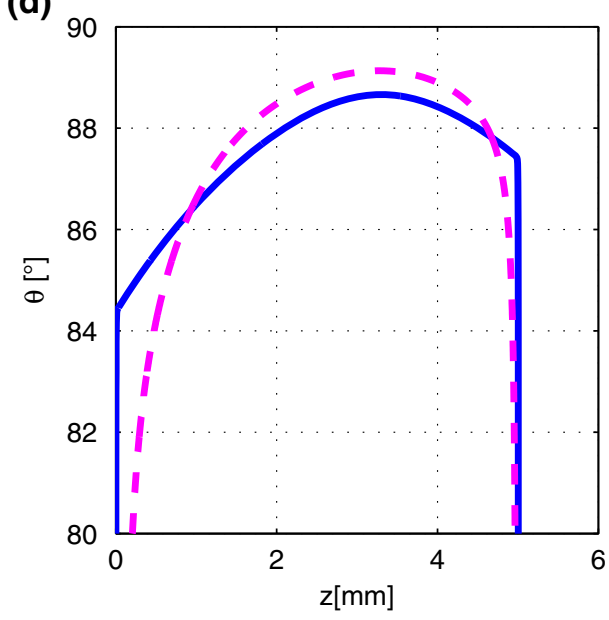

(b)

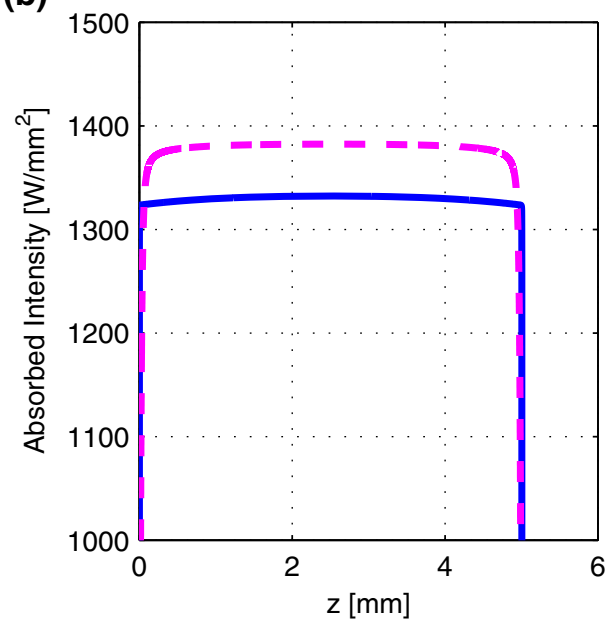

Fig. 10 Absorbed intensity profiles for a 5-mm-thick $90 \mathrm{MnCrV} 8$ workpiece.

a Absorbed intensity profiles for a focal position $f / d=-0.33$.

Magenta pecked curve $\lambda=10.6$ $\mu \mathrm{m}$, blue solid curve $\lambda=1.03$ $\mu \mathrm{m}$. b Absorbed intensity profiles for a focal position $f / d=-0.50$. Magenta pecked curve $\lambda=10.6 \mu \mathrm{m}$, blue solid curve $\lambda=1.03 \mu \mathrm{m}$ to point out that, despite the $\mathrm{CO}_{2}$-laser absorptivity being higher than the disk laser absorptivity for most parts of the cutting profile, the corresponding absorbed intensity is higher for the disk laser along the entire cutting profile. This is the main reason why the cutting 
speed of the disk laser is higher than the cutting speed of the $\mathrm{CO}_{2}$-laser. For a focal position around the middle of the workpiece the situation is reversed for thicknesses in the range of $5-10 \mathrm{~mm}$ (see Figs. 6a, b, $10 \mathrm{~b})$. It is worth noting that the absorbed intensity, as shown in Fig. 10, is constant along the entire cutting profile. This, however, is not surprising since we analyzed the stationary solutions and stationary implies that the same amount of material must be removed per unit time at every point along the cutting profile. Finally, the results for the basic and the extended model show virtually identical cutting profiles, absorptivity profiles, and absorbed intensity profiles.

\section{Conclusions}

Based on a local energy balance equation, we presented a simple derivation of the Niziev equation and extended it by dropping one of the most questionable initial assumptions, namely the absence of heat conduction to the bulk. In order to solve the nonlinear partial differential equation of the first order, we split it in a stationary and a time-dependent part. By applying the method of characteristics, we transformed the partial differential equation for the stationary part to a system of ordinary differential equations. This was subsequently solved numerically and we presented a procedure to monitor the accuracy of the numerical solution. Next, numerical solutions were computed for the same material and laser cutting parameters as used in reference [1] and the main findings are:

- Generally, we find a qualitative agreement between the basic model and experimental results. On an absolute scale, however, no-negligible discrepancies are observed.

- When employing the extended model, which incorporates heat conduction through an approximate analytic expression, we find excellent quantitative agreement between theory and experiment. The heat conduction losses to the bulk material can be as high as $90 \%$ of the absorbed intensities and, therefore, must not be neglected. Moreover, the values calculated for the conductive losses are in good agreement with results obtained by other methods.

- By analyzing the general features of the absorptivity curves and, more importantly, the absorbed intensities along the cutting profiles we find clear indications why the disk laser performs better than the $\mathrm{CO}_{2}$ laser in a large parameter range. The energy transfer is more effective for the disk laser.
For the near future, we plan a more detailed comparison of the full 3D model with experimental results. Also, we currently are refining the model by incorporating the waveguide properties of the kerf and to allow for a process temperature higher than the melting temperature.

Acknowledgments The authors are indebted to B. Neuenschwander, R. Augsburger, and V. Romano for helpful discussions and the Swiss KTI for financial support.

\section{References}

1. L.D. Scintilla, L. Tricarico, A. Mahrle, A. Wetzig, E. Beyer, A comparative study of cut front profiles and absorptivity behavior for disk and $\mathrm{CO}_{2}$ laser beam inert gas fusion cutting. J. Laser Appl. 24(5), 052006-1 (2012)

2. K. Hirano, R. Fabbro, Possible explanations for different surface quality in laser cutting with 1 and $10 \mu \mathrm{m}$ beams. J. Laser Appl. 24(1), 012006-1 (2012)

3. M. Vicanek, G. Simon, H.M. Urbassek, I. Decker, Hydrodynamical instability of melt flow in laser cutting. J. Phys. D: Appl. Phys. 20, 140-145 (1987)

4. C. Wandera, A. Salminen, V. Kujanpaa, Inert gas cutting of thick-section stainless and medium-section aluminum using a high power fiber laser. J. Laser Appl. 21(3), 154-161 (2009)

5. C. Wandera, V. Kujanpaa, Characterization of the melt removal rate in laser cutting of thick-section stainless steel. J. Laser Appl. 22(2), 62-70 (2010)

6. M. Vicanek, G. Simon, Momentum and heat transfer of an inert gas jet to the melt in laser cutting. J. Phys. D: Appl. Phys. 20, 1191-1196 (1987)

7. M. Sparkes, M. Gross, S. Celotto, T. Zhang, and W. O'Neill, Inert cutting of medium section stainless steel using a $2.2 \mathrm{kw}$ high brightness fibre laser. In ICALEO 2006 Congress Proceedings, pp. 197-205, Orlando, FL, 2006. Laser Institute of America. Paper No. 402.

8. A .Mahrle, E. Beyer, Theoretical aspects of fibre laser cutting. J. Phys. D: Appl. Phys. 42, 175597 (2009)

9. F. O. Olsen, Laser cutting from $\mathrm{CO}_{2}$ laser to disc or fiber laserPossibilities and challenges. In ICALEO 2011 Congress Proceedings, pp. 6-15, Orlando, FL, 2011. Laser Institute of America. Paper No. 101.

10. A. Riveiro, F. Quintero, F. Lusquiños, J. Pou, A. Salminen, V. Kujanpaa. Influence of assist gas in fibre laser cutting of aluminum-copper alloy. In ICALEO 2008 Congress Proceedings, pp. 688-694, Orlando, FL, 2008. Laser Institute of America. Paper No. 2004.

11. L.D. Scintilla, L. Tricarico, Estimating cutting front temperature difference in disk and $\mathrm{CO}_{2}$ laser beam fusion cutting. Optics Laser Technol. 44, 1468-1479 (2012)

12. D. Petring, T. Molitor, F. Schneider, N. Wolf, Diagnostics, modeling and simulation: three keys towards mastering the cutting process with fiber, disk and diode lasers. Phys. Procedia 39, 186-196 (2012)

13. V.G. Niziev, Theory of CW laser beam cutting. Laser Phys. 3(3), 629 (1993)

14. V. G. Niziev, A. V. Nesterov, Influence of beam polarization on laser cutting efficiency. J. Phys. D: Appl. Phys. 32, 1455, (1999)

15. M. Born, E. Wolf, Principles of optics, Cambridge University Press, The Edinburgh Building, Cambridge CB2 2RU, UK, Sixth edition, (1997) 
16. J. A. Stratton, Electromagnetic theory, McGraw-Hill Book Company, Inc., New York and London, (1941)

17. A. Kaplan, Theoretical analysis of laser cutting. Shaker Verlag, Aachen, (2002)

18. W. Schulz, D. Becker, J. Franke, R. Kammerling, G. Herziger, Heat conduction losses in laser cutting of metals. J. Phys. D: Appl. Phys. 26, 1357-1363 (1993)

19. R. Courant, D. Hilbert, Methods of Mathematical Physics, vol. 2, Interscience Publishers, New York, (1962)

20. P.A. Bélanger, C. Paré, Optical resonators using graded-phase mirrors. Optics Lett. 16(14), 1057-1059 (1991)

21. P. Atanasov, Some aspects of high pressure $\mathrm{N}_{2}$-assisted $\mathrm{CO}_{2}$ laser cutting of metals. In Proc. SPIE 1810, 9th International Symposium on Gas Flow and Chemical Lasers, vol. 1810, pp. 628-631, May 1993
22. M. Dell'Erba, G. Daurelio, M. Ferrara, $\mathrm{CO}_{2}$ laser cutting process of thick aluminium. Appl. Phys. Commun. 5(1-2), 23-35 (1985)

23. G. Daurelio, M. Dell'Erba, L. Cento, Cutting copper sheets by $\mathrm{CO}_{2}$ laser. Lasers Appl. pp. 59-64 (1986)

24. S. Stelzer, A. Mahrle, A. Wetzig, E. Beyer, Experimental investigations on fusion cutting stainless steel with fiber and $\mathrm{CO}_{2}$ laser beams. Phys. Procedia 41, 392-397 (2013)

25. L.D. Scintilla, L. Tricarico, A. Wetzig, E. Beyer Investigation on disk and $\mathrm{CO}_{2}$ laser beam fusion cutting differences based on power balance equation. Int. J. Mach. Tools Manuf. 69, 30-37 (2013)

26. L.D. Scintilla, L. Tricarico, A. Wetzig, A. Mahrle, E. Beyer, Primary losses in disk and $\mathrm{CO}_{2}$ laser beam inert gas fusion cutting. J. Mater. Process. Technol. 211, 2050-2061 (2011) 